Research Article

\title{
Deep Learning-Based Three-dimensional Transvaginal Ultrasound in Diagnosis of Intrauterine Adhesion
}

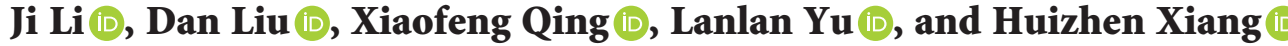 \\ Department of Gynecology, Huaihua First People’s Hospital, Huaihua 418000, Hunan, China \\ Correspondence should be addressed to Huizhen Xiang; 15110100012@pop.zjgsu.edu.cn
}

Received 8 September 2021; Revised 5 November 2021; Accepted 6 November 2021; Published 28 November 2021

Academic Editor: M Pallikonda Rajasekaran

Copyright (C) $2021 \mathrm{Ji} \mathrm{Li}$ et al. This is an open access article distributed under the Creative Commons Attribution License, which permits unrestricted use, distribution, and reproduction in any medium, provided the original work is properly cited.

\begin{abstract}
This study was aimed to enhance and detect the characteristics of three-dimensional transvaginal ultrasound images based on the partial differential algorithm and HSegNet algorithm under deep learning. Thereby, the effect of quantitative parameter values of optimized three-dimensional ultrasound image was analyzed on the diagnosis and evaluation of intrauterine adhesions. Specifically, 75 patients with suspected intrauterine adhesion in hospital who underwent the hysteroscopic diagnosis were selected as the research subjects. The three-dimensional transvaginal ultrasound image was enhanced and optimized by the partial differential equation algorithm and processed by the deep learning algorithm. Subsequently, three-dimensional transvaginal ultrasound examinations were performed on the study subjects that met the standards. The March classification method was used to classify the patients with intrauterine adhesion. Finally, the results by the three-dimensional transvaginal ultrasound were compared with the diagnosis results in hysteroscope surgery. The results showed that the HSegNet algorithm model realized the automatic labeling of intrauterine adhesion in the transvaginal ultrasound image and the final accuracy coefficient was $97.3 \%$. It suggested that the three-dimensional transvaginal ultrasound diagnosis based on deep learning was efficient and accurate. The accuracy of the three-dimensional transvaginal ultrasound was $97.14 \%$, the sensitivity was $96.6 \%$, and the specificity was $72 \%$. In conclusion, the three-dimensional transvaginal examination can effectively improve the diagnostic efficiency of intrauterine adhesion, providing theoretical support for the subsequent diagnosis and grading of intrauterine adhesion.
\end{abstract}

\section{Introduction}

Intrauterine adhesion is a common gynecological disease, also known as Asherman syndrome in clinical practice. Its symptoms were first described by Fritsch in 1894 and were not reported in detail by Asherman for the first time until 1948 [1-4]. The main reason for intrauterine adhesion is the trauma of the pregnant or nonpregnant uterus, resulting in damage to the base of the endometrium and then partial occlusion of the uterine cavity. The intrauterine adhesions caused by nonpregnancy only accounted for $9 \%$ of the total [5]. Intrauterine adhesion in different parts and of different degrees differs in clinical manifestations, but the essence of intrauterine adhesion is the fibrosis of the endometrium.

In recent years, the incidence of intrauterine adhesion has increased significantly due to the annual increase in abortion and medical abortion. In China, intrauterine adhesion has become the second leading cause of female infertility following fallopian tube factors. Studies have shown that the incidence of intrauterine adhesion is as high as $25 \% \sim 30 \%$ after multiple abortions and curettage after a miscarriage can cause $[6,7]$. The symptoms such as abdominal pain, reduced menstruation, and infertility caused by intrauterine adhesion are extremely difficult to treat and the recurrence rate is high, seriously threatening women's reproductive health and mental health [8]. Intrauterine adhesion is generally divided into mild, moderate, and severe stages. Intrauterine adhesion in different stages has different treatment options and prognoses. For mild intrauterine adhesion, equipment blunt separation or uterine cavity liquid injection expansion program are mainly used and good curative effects and prognostic effects have been realized; for moderate intrauterine adhesion, hand hysteroscopy is performed for deadhesion and postoperative 
estrogen adjuvant treatment is required; for severe intrauterine adhesion, hysteroscopic intrauterine adhesion separation is required. However, there is a high probability of complications and perforation bleeding during the operation, the postoperative prognosis is not optimistic, and the probability of a successful pregnancy is only $22.5 \% \sim 33.3 \%$ $[9,10]$. Therefore, the early diagnosis and early treatment of intrauterine adhesion are of great significance. Correct diagnosis and staging evaluation can provide an effective basis for the diagnosis and treatment of intrauterine adhesion.

Currently, the main diagnostic methods for intrauterine adhesion include hysteroscopy and transvaginal ultrasound. Hysteroscopy has been clinically determined to be the gold standard for the diagnosis and treatment of such diseases [11]. It has high specificity and enables the doctor to observe the characteristics of the uterine cavity under direct vision so that the location and range of intrauterine adhesion can be accurately and effectively evaluated, which is an important basis for the prognosis of subsequent treatment. However, hysteroscopy is invasive, which will affect the patient's body and spirit and has high requirements on the experience of the operator. At the same time, there exists the risk of perforation bleeding during the operation. Therefore, its applications are limited in the clinic. The vaginal three-dimensional ultrasound examination is a widely used clinical imaging method. In addition to intrauterine adhesion, it has high specificity for a variety of gynecological diseases. It is characterized by simple operation, noninvasiveness, and low examination cost $[12,13]$ and is thus generally accepted by patients. Nevertheless, in practice, factors, such as physical movement, special examination parts, heartbeat, and breathing, will cause blur, artifacts, and noise in the image. Deep learning has been widely used in search, data mining, natural language processing, and image analysis [14].

The image restoration method based on deep learning can restore the damaged image well and the restored image contains rich image feature information. In the process of transmitting and acquiring digital images, the signal may change due to the imaging principle, sensor defects, imperfect instruments, changes in the surrounding environment, various other human factors, etc. In addition, transmission errors and image compression will cause noise, which will pollute the original image, blur the image information, and reduce the image quality. It affects the observation, processing, understanding, and use of image information, and ultimately the image will lose the basic function and significance of information storage. The subsequent processing of the image will also be affected. Thus, it has become one of the key steps in image and video processing to denoise for the image quality improvement. The noise and detail texture are in the high-frequency area of the image, but most of the image information is stored in the edge part. In the process of denoising, the texture information will be mistakenly filtered out as being considered to be the noise, which will result in blur for image information loss. Therefore, there is an irreconcilable contradiction between detail texture information protection and image denoising. The goal of image denoising is to maintain the edge and texture detail information as much as possible while removing noise. The traditional image denoising algorithm cannot balance the contradiction, but the partial image denoising algorithm under deep learning could achieve the selective smooth to balance the contradiction. As a result, it can directly reflect the degree of cervical adhesion and provide important parameters such as the volume of the endometrium in the uterine cavity.

In this study, the partial differential algorithm and HSegNet algorithm under deep learning were applied for the processing of three-dimensional transvaginal ultrasound images. As the diagnosis of intrauterine adhesions was done based on it, its application effects were evaluated, which provided reference for the improvement of the clinical diagnosis of intrauterine adhesions.

\section{Materials and Methods}

2.1. Research Subject. Seventy-five patients with suspected intrauterine adhesion in hospital who underwent hysteroscopic diagnosis were selected as the research subjects. They were between 24-45 years old and the average age was $36.4 \pm 2.3$ years old. All patients did not have other diseases on the uterine cavity such as uterine cavity polyps and submucosal fibroids.

Inclusion criteria: (I) patients with clinical symptoms related to intrauterine adhesion, (II) routine ultrasound examinations confirmed that the patient had no other uterine cavity diseases, (III) patients with infertility, oligomenorrhea, and other problems requiring hysteroscopic surgery, and (IV) patients who have signed an informed consent form for treatment.

Exclusion criteria: patients who cannot undergo hysteroscopic surgery for other reasons.

\subsection{Three-Dimensional Transvaginal Ultrasound} Examination. The specific steps were as follows. Step (I): before the examination, the patient needed to empty the urine in the bladder. Step (II): the patient was in the lithotomy position. RIC5-9H transvaginal probe was disinfected with an iodine cotton ball and an appropriate amount of couplant was squeezed into the condom which was put on the probe. Step (III): the operator put on the gloves, told the patient to relax, held the probe handle in the right hand, and slowly sent the probe into the vaginal vault. Step (IV): the pelvic cavity was scanned layer by layer using basic techniques such as rotation, tilting, and pumping to observe the general condition of the uterus and appendages, and then, the diagnosis was determined by color Doppler ultrasound apparatus. Step (V): if the position of the uterus is high, the operator can use the left hand to gently press the lower abdomen for better display.

\subsection{Hysteroscope Examination Methods. The patients knew} clearly the experiment and singed the informed consents after sufficient preoperative consultation. Before the surgery, the patients had an absolute diet; the vulva, vagina, and cervix were disinfected with covering disinfection towels, in the bladder lithotomy position. The anterior lip of the cervix 
was clamped with cervical forceps to explore the depth of the uterine cavity, and the cervix was expanded to a half size larger than the diameter of the outer sheath of the hysteroscope. The hysteroscope was connected to the television camera, light source, and uterine dilation system. The air bubbles in the uterine dilation fluid were drained out, and the hysteroscope was put into the uterine cavity slowly while swelling the uterus. The uterine cavity was inspected in detail, in the order of the fundus, the uterus walls, the corners, the ostium of the fallopian tube, the internal cervix, and the cervical canal. If a viable tissue needed to be taken, the microforceps was used to locate and pick the sample through the operating hole.

The general condition of the patients must be concerned all the time during the surgery. When there was shortness of breath or coughing, the surgery was stopped immediately; meanwhile, the gas was released and the symptomatic treatment was given. When the cervical canal of patients was tight, forcible expansion was inadvisable to prevent bleeding from the rupture of the cervical canal. The examination time was preferably within 5 minutes. Injury in the uterine cavity was avoided; otherwise, there would be a risk of air embolism.

2.4. Staging Criteria of Intrauterine Adhesion. Nowadays, there are three main clinical classification and scoring standards for intrauterine adhesion $[15,16]$. In this study, the AFS classification method by the American Reproductive Association is mainly used and the March classification method is used as the basis for three-dimensional ultrasound classification evaluation.

As per the AFS classification, the narrow range of the uterine cavity $<1 / 3$ scores 1 , with $1 / 3 \sim 2 / 3$ scoring 2 and $>2 /$ 3 scoring 4 . According to the type of adhesion, 1 point was for film-like adhesions, adhesion between the thin film and dense scores 2 points, and dense adhesion scores 4 points. According to menstrual condition, menstrual increase scores 0 , minor menstruation scores 2 points, and amenorrhea scores 4 points. $1-4$ points are considered mild; 5-8 points are considered moderate; $9-12$ points are considered severe.

2.5. Differential Equation Algorithm to Restore Transvaginal Ultrasound Images. The overall variational image restoration model (TV model) in the differential equation algorithm has excellent anisotropic diffusion. It can also perform denoising while restoring the image, and it is relatively simple in terms of calculation and solution.

Assuming that the restored image is $F=\mathrm{R} \cup \mathrm{E}$, the cost function of the image to be restored is expressed as follows:

$$
T(f)=\int_{f} r\left(\left|\nabla_{f}\right|\right) d x d y .
$$

At the edge of the area that needs to be repaired, there is an impact function. At this time $\nabla_{f}$. Then, the standby function needs to satisfy the following conditions:

$$
\int_{f} r\left(\left|\nabla_{f}\right|\right) d x d y<\infty
$$

In the differential equation algorithm model, $r\left(\left|\nabla_{f}\right|\right)=r\left(\left|\nabla_{f}\right|\right)$. Then, the function at this time is expressed as follows:

$$
T(f)=\int_{f}\left|\nabla_{f}\right| d x d y .
$$

If denoising is performed in the process of restoring the image, the following conditions must be met:

$$
\delta^{2}=\frac{1}{s(E)_{E}} \int_{E}\left|f-f_{0}\right|^{2} d x d y,
$$

where $\delta^{2}$ is the variance of Gaussian noise, $s(E)$ represents the undamaged part of the image, $f$ is the restored image, and $f_{0}$ is the original image with noise. Combining equations (3) and (4), we can obtain the overall energy functional function:

$$
G_{\lambda}(f)=\int_{f}\left|\nabla_{f}\right| d x d y+\frac{\lambda}{2} \int_{E}\left|f-f_{0}\right|^{2} d x d y,
$$

where $\lambda$ represents the Lagrange multiplier. Next, the minimum value of the energy functional function of this model is solved:

$$
E(f)=\int_{w} D\left(x, y, f, \frac{\phi_{f}}{\phi x}, \frac{\Phi_{f}}{\phi y}\right) d x d y .
$$

To take the minimum value, the following conditions should be met:

$$
D_{f}-\frac{\phi}{\phi J C} D_{f_{x}}-\frac{\phi}{\phi y} D_{f_{y}}=0
$$

In the TV model in the differential algorithm equation,

$$
D\left(x, y, f, \frac{\phi_{x}}{\phi n}, \frac{\phi_{y}}{\phi n}\right)=\sqrt{\left(\frac{\varphi_{f}}{\varphi_{x}}\right)^{2}+\left(\frac{\varphi f}{\varphi y}\right)^{2}} \frac{\lambda}{2}\left(f-f_{0}\right)^{2} \text {. }
$$

Combining equations (8) and (7), we can obtain the minimum energy functional equation of the TV model:

$$
-\nabla \cdot\left(\frac{\nabla_{f}}{\left|\nabla_{f}\right|}\right)+\lambda\left(f-f_{0}\right)=0 .
$$

For a certain point $z=(x, y) \in \omega$ on the image, the Lagrange multiplier in equation (9) satisfies the following conditions:

$$
\lambda= \begin{cases}\lambda, & z \in E \\ O, & z \in D .\end{cases}
$$

2.6. HSegNet Algorithm for Marking Intrauterine Adhesion in Transvaginal Ultrasound Images. In this study, the 
HSegNet algorithm is used to judge and label the intrauterine adhesion parts in the ultrasound image. The HSegNet algorithm is based on a deep learning framework and consists of a two-level network, using an automatic context model [12]. The overall framework is shown in Figure 1.

The HSegNet algorithm takes a three-dimensional transvaginal ultrasound image as input, uses the encoder part of the network model to encode it to obtain high-dimensional features, and then uses the existing doctor marks for learning. The HSegNet algorithm is a typical deep learning convolutional neural network (CNN). In the HSegNet algorithm, we need to correct the error value obtained in the calculation using the cross-entropy loss function Softmax. If $F_{x_{i}}$ is the output of $x_{i}$, then the crossentropy loss function is expressed as follows:

$$
F_{x_{i}}=\frac{e^{x_{i}}}{\sum_{c=1}^{a} e^{x_{i}}} .
$$

Next, the cross entropy of each pixel is further calculated as follows:

$$
T(i)=\sum_{a=1}^{a} y_{i, a} \log \left(d_{i}, a\right),
$$

where $T(i)$ represents the cross entropy of the $i$ th pixel, $y_{i, a}$ represents the true label of the $i$ th pixel, and $d_{i}$ and $a$ represent the probability of the $i$ th pixel. Then, the final loss cost function is expressed as follows:

$$
\text { Loss }=\frac{1}{E \times F \times p} \sum_{i=1}^{E \times F \times P} T(i) \text {. }
$$

2.7. Statistical Standards. SPSS19.0 software was used for statistical analysis. Measurement data conforming to normal distribution were expressed as mean \pm standard deviation and comparisons between groups were realized by independent sample $t$-test; measurement data that did not conform to normal distribution were expressed by the median value and four-point position representation, and the nonparametric rank-sum test was used to analyze the difference between groups. Count data were expressed by $n$ (\%) and the comparison of differences between groups was analyzed by the chi-square test. $P<0.05$ indicated that the difference was statistically significant.

\section{Results}

3.1. The Repair Effects of the Differential Equation Algorithm on the Three-Dimensional Transvaginal Ultrasound Image. The differential equation model image restoration algorithm of deep learning was used to optimize the undersampled three-dimensional transvaginal ultrasound image. As shown in Figure 2, the undersampled three-dimensional transvaginal ultrasound image contained more noise and blur. After the image was reconstructed by the differential equation algorithm model, the structure was clearer.
3.2. The Effectiveness of HSegNet Algorithm Model to Label the Transvaginal Ultrasound Image. In this study, the HSegNet algorithm model was used to realize the automatic labeling of intrauterine adhesion in the transvaginal ultrasound image. To solve the serious fuzzy shadow problem in the three-dimensional ultrasound image, a differential equation algorithm was used to repair and enhance the original image, and the automatic context model was used to improve the final automatic labeling accuracy. After comparison with the manual labeling by the doctor, the algorithm was found to reach an accuracy rate of $97.3 \%$. Figure 3 was the schematic diagram of automatic labeling, and Figure 4 was the manual labeling by the doctor.

\subsection{Three-Dimensional Transvaginal Ultrasound Diagnosis} and Hysteroscopy Diagnosis Results. Three-dimensional transvaginal ultrasound imaging and hysteroscopy were performed on 75 patients included in the study, and the diagnosis results were compared and analyzed. It was found that 68 of the 75 patients were judged as intrauterine adhesion through three-dimensional ultrasound. Compared with the diagnosis results of 70 cases of hysteroscopy, the accuracy of three-dimensional transvaginal ultrasound images was $97.14 \%$, the sensitivity was $96.6 \%$, and the specificity was $72 \%$. As for the degree of intrauterine adhesion for 75 patients, there were 39 cases of mild patients, accounting for $57.35 \%, 20$ cases of moderate patients, accounting for $29.41 \%$, and 9 cases of severe patients, accounting for $13.23 \%$. As shown in Figure 5, there was no statistical difference in the diagnosis rates $(P>0.05)$ of the two methods and the accuracy of threedimensional transvaginal ultrasound alignment was $100 \%$ in the diagnosis of moderate and severe intrauterine adhesion.

3.4. Analysis of the Value of Each Parameter of Three-Dimensional Transvaginal Ultrasound in the Diagnosis of Intrauterine Adhesion. After the diagnosis by threedimensional transvaginal ultrasound imaging on 75 patients included in this study, several indexes of endometrial volume (V) and endometrial blood flow parameters (VI, FI, VFI) were collected. The average endometrial volume of 7 subjects with normal uterine cavity was $4.13 \pm 0.66 \mathrm{~cm}^{3}$, and the average endometrial blood flow parameters (VI, FI, VFI) were $4.25 \pm 1.30 \%, 27.76 \pm 4.87$, and $1.37 \pm 0.62$, respectively. There were 39 patients with mild intrauterine adhesion. The mean endometrial volume was $2.38 \pm 0.74 \mathrm{~cm}^{3}$, and the mean endometrial blood flow parameters (VI, FI, VFI) were $3.47 \pm 1.19 \%, 24.69 \pm 4.13$, and $0.73 \pm 0.37$, respectively. There were 20 patients with moderate intrauterine adhesion. The average endometrial volume was $1.73 \pm 0.54 \mathrm{~cm}^{3}$, and the average endometrial blood flow parameters (VI, FI, VFI) were $2.47 \pm 0.59 \%$, $20.26 \pm 4.83$, and $0.53 \pm 0.29$, respectively. There were 9 patients with severe intrauterine adhesion. The mean endometrial volume was $1.12 \pm 0.34 \mathrm{~cm}^{3}$, and the mean endometrial blood flow parameters (VI, FI, VFI) were $1.47 \pm 0.39 \%, 16.56 \pm 1.13$, and $0.25 \pm 0.57$, respectively. 


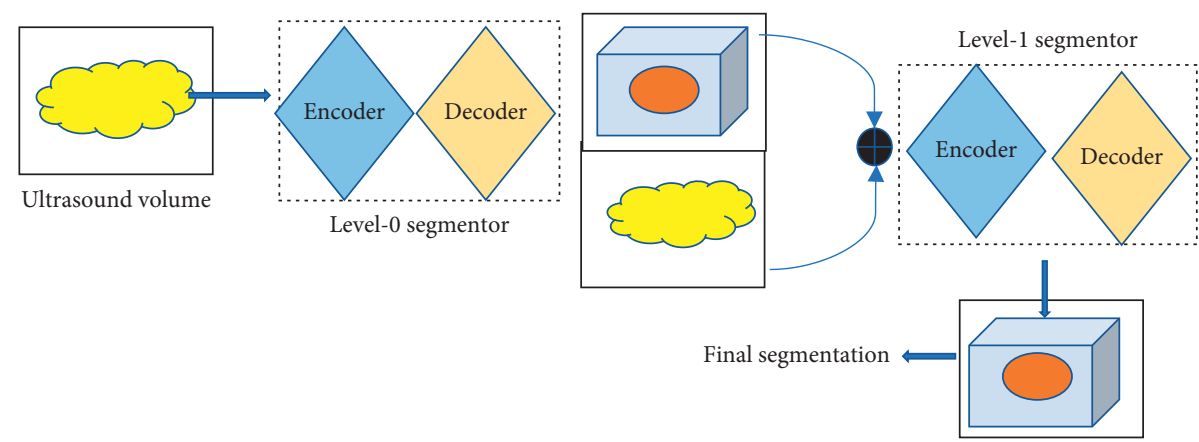

FiguRe 1: The overall framework of the HSegNet algorithm.
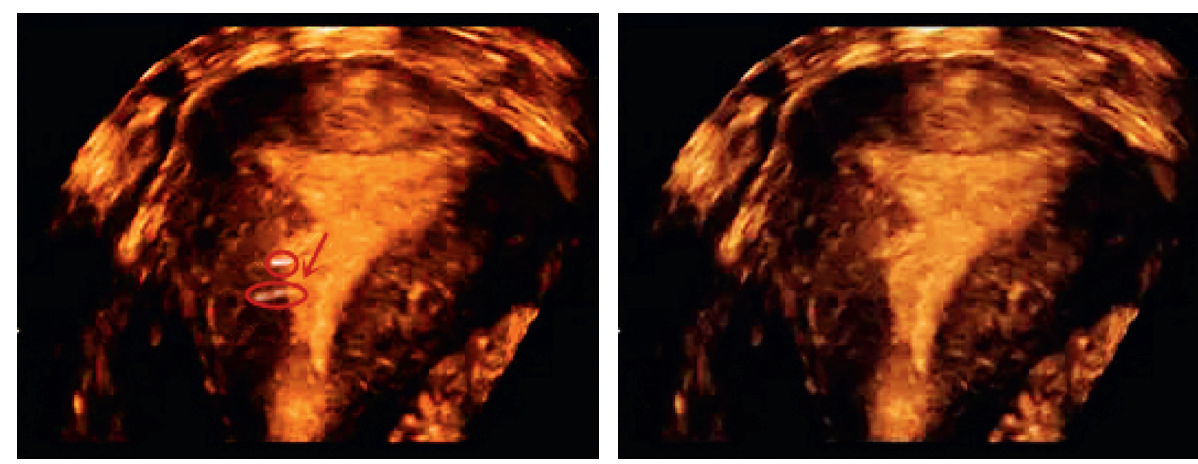

FigURE 2: Differential equation model for three-dimensional transvaginal ultrasound image restoration.
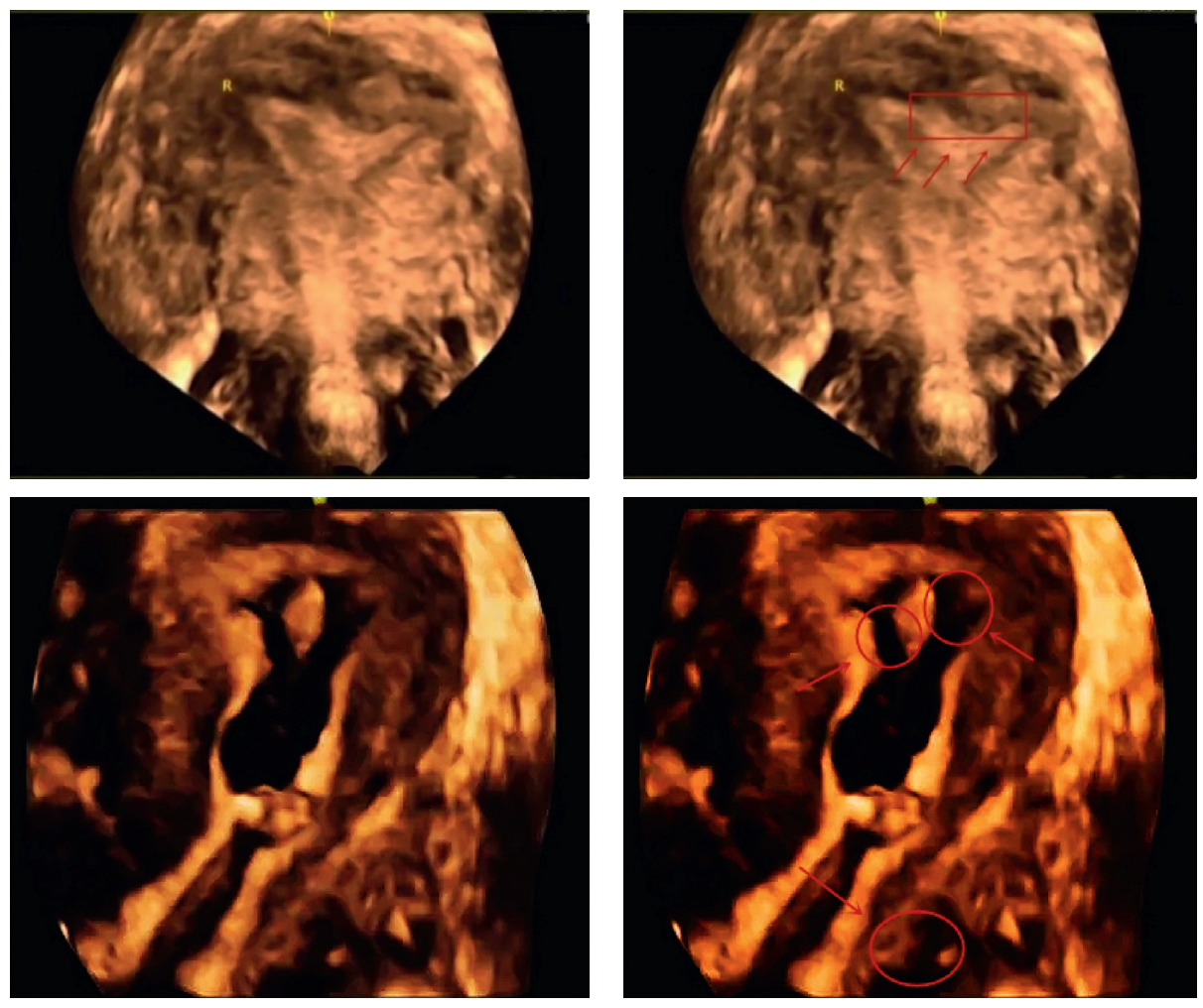

(a)

(b)

FIGURE 3: Schematic diagram of automatic labeling by the HSegNet algorithm model ((a) unlabeled and (b) automatically labeled intrauterine adhesion). 


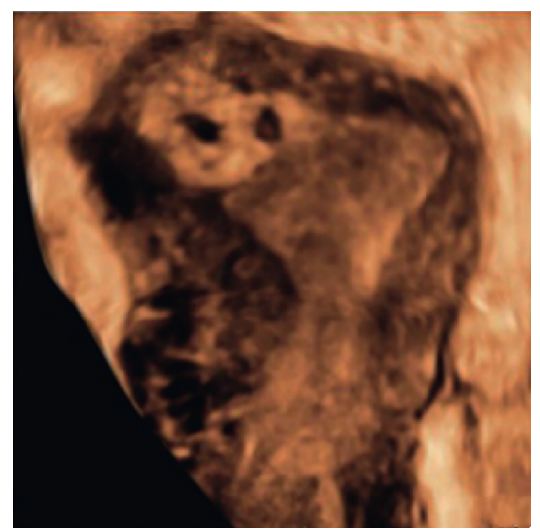

(a)

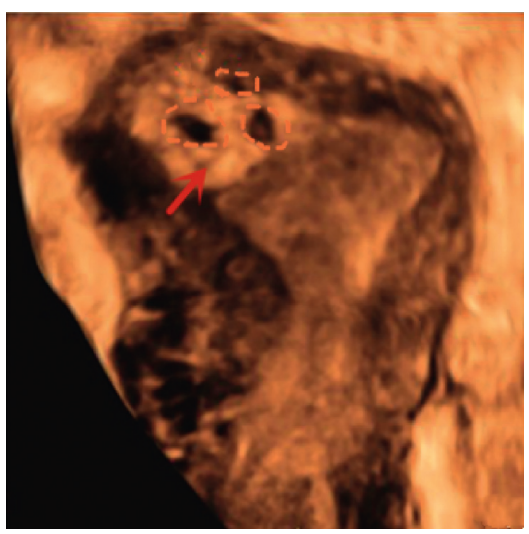

(b)

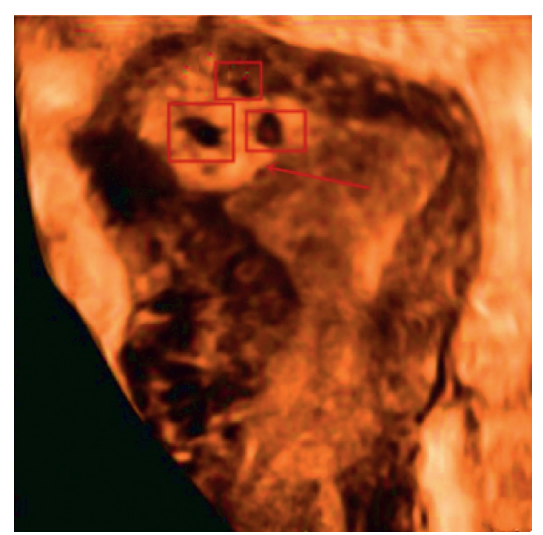

(c)

Figure 4: Comparison of automatic labeling by the HSegNet algorithm model and manual labeling ((a) unlabeled, (b) manual labeling by the doctor, and (c) the automatic labeling by the algorithm).

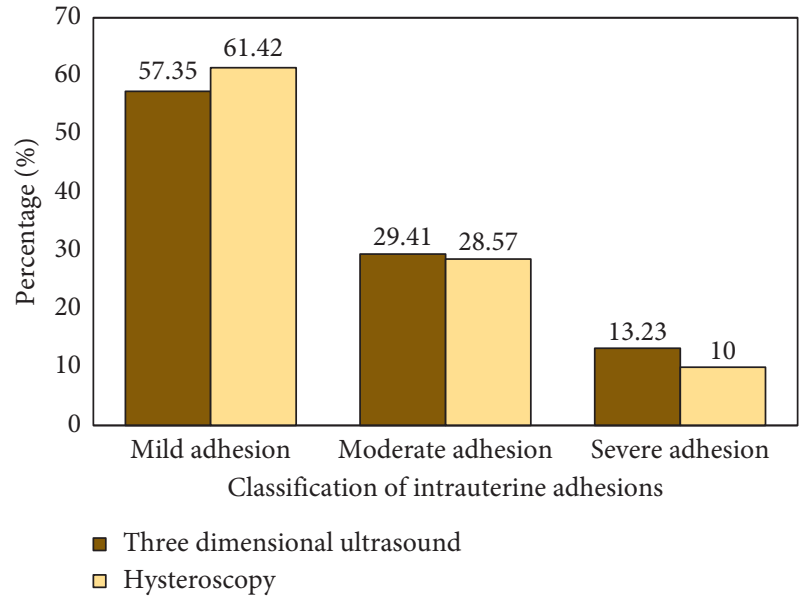

FIGURE 5: Three-dimensional transvaginal ultrasound diagnosis and hysteroscopy diagnosis results and intrauterine adhesion classification.

The parameters of different degrees of intrauterine adhesion are shown in Figures 6 and 7.

3.5. Accuracy of the Three-Dimensional Transvaginal Ultrasound Quantitative Measurement to Diagnose the Intrauterine Adhesion. The accuracy of the three-dimensional transvaginal ultrasound quantitative measurement was evaluated based on the endometrial volume $(\mathrm{V})$ and endometrial blood flow parameters (VI, FI, VFI) parameters. Figure 8 shows the specific results.

\section{Discussion}

Intrauterine adhesion is a common stubborn gynecological disease, also known as Ashman syndrome, arising from the partial or complete occlusion of the uterine cavity due to the damage of the endometrium. In general, infection or improper abortion surgery may cause damage to the endometrium, which will cause the damaged endometrium to adhere to each other. The main clinical symptoms include

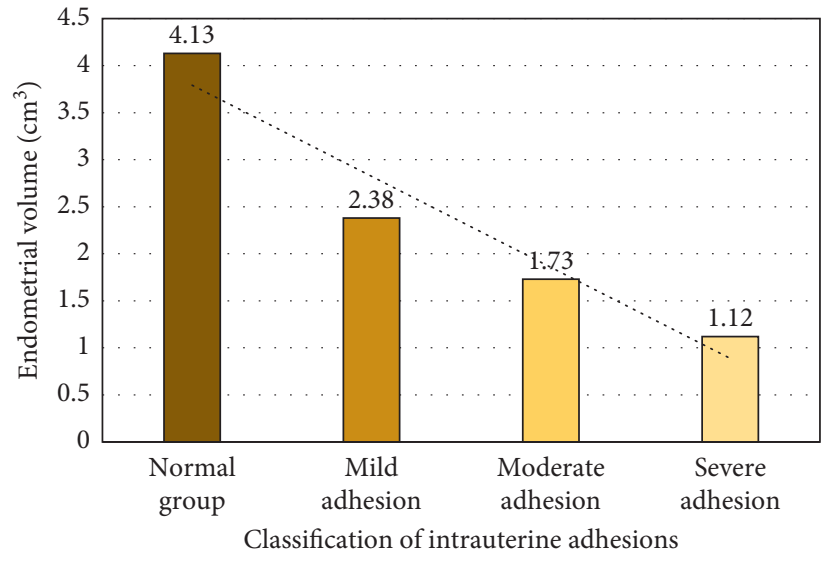

FIgURE 6: The endometrial volume of different degrees of intrauterine adhesion.

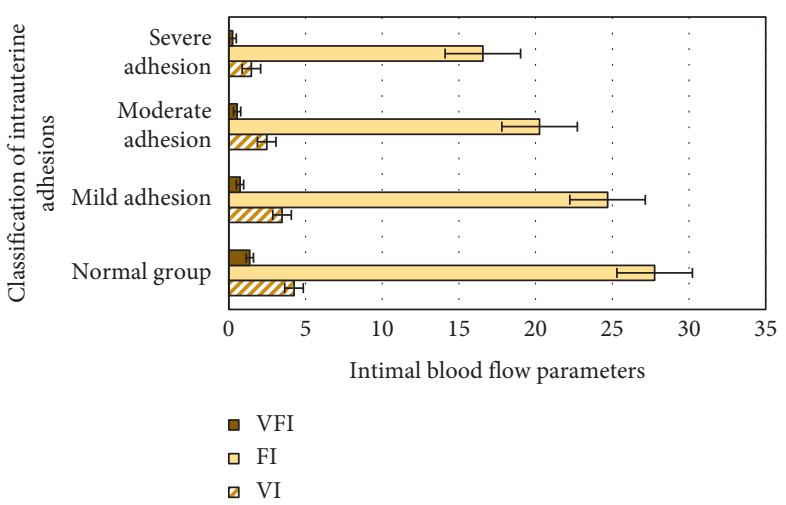

FIGURE 7: The intimal blood flow parameters of different degrees of intrauterine adhesion.

abnormal menstruation or amenorrhea, infertility, and abdomen pain. At present, ultrasound examination is mainly used to diagnose intrauterine adhesion in clinical practice because of its convenient operation, noninvasiveness, low 


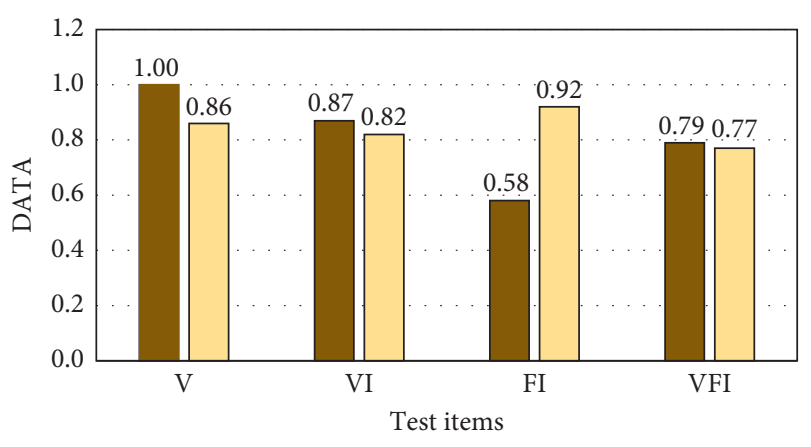

口 Specificity Sensitivity

FIgURE 8: Accuracy of the three-dimensional transvaginal ultrasound quantitative measurement.

price, and high repeatability. In the past, two-dimensional examinations were often used in clinical practice. Nowadays, three-dimensional ultrasound is mostly used. Three-dimensional ultrasound examinations have precise electronically controlled probes and three-dimensional image acquisition programs, which can achieve the automatic acquisition of three-dimensional images [17].

The traditional two-dimensional transvaginal ultrasound can accurately measure the thickness of the patient's endometrium and can observe the echo changes of the endometrium and intrauterine effusion. However, the overall shape of the uterine cavity cannot be judged. In the diagnosis of intrauterine adhesion, traditional two-dimensional ultrasonography has great limitations, and it is prone to missed diagnoses and misdiagnoses [18]. As the intrauterine adhesions in 75 patients were graded, the mild patients accounted for $57.35 \%$, moderate patients accounted for $29.41 \%$, and severe ones accounted for $13.23 \%$. According to hysteroscopy diagnosis, there were $61.42 \%$ of mild patients, $28.57 \%$ of moderate patients, and $13.23 \%$ of severe patients. The results of three-dimensional transvaginal ultrasound and hysteroscopy and the grades of intrauterine adhesions are shown in Figure 5, and there was no statistical difference between the diagnosis rates under the two methods $(P>0.05)$. The transvaginal three-dimensional ultrasound examination makes it possible to observe the coronal section that is difficult to detect by two-dimensional ultrasound when diagnosing patients with intrauterine adhesion. Hence, it makes up for the limitations of two-dimensional ultrasound. In addition, three-dimensional ultrasound can effectively observe the patient's uterus and the anatomical relationship and location of the lesion so that the doctor can observe the endometrium at various angles, which greatly reduces the occurrence of missed diagnoses and misdiagnoses. The automatic labeling of the intrauterine adhesions was achieved in the transvaginal ultrasound images under the HSegNet algorithm model, with the final accuracy coefficient of $97.3 \%$. It also had a good consistency with the doctors' manual labeling. It was suggested that the threedimensional transvaginal ultrasound image diagnosis under deep learning was efficient and accurate currently. The accuracy of transvaginal three-dimensional ultrasound imaging was $97.14 \%$, together with a sensitivity of $96.6 \%$ and a specificity of $72 \%$.

At present, the main classification methods for intrauterine adhesion include the European Society of Gynecological Endoscopy SGE classification [19], the American Society of Reproductive Society AFS classification, and March classification. They all divide the intrauterine adhesion into three levels: mild, moderate, and severe according to the scope and characteristics of intrauterine adhesion. There has not been a unified classification standard, but March classification is often used in China. The precise grading of intrauterine adhesion can effectively improve the clinical treatment effects of intrauterine adhesion. Clinically, three-dimensional transvaginal ultrasound mainly judges the specific conditions of the uterine cavity by observing data such as endometrial thickness, endometrial echo, intrauterine adhesion site, and adhesion range. Compared with traditional two-dimensional ultrasound, three-dimensional transvaginal ultrasound has obvious advantages in observing the scope and details of the surface in the uterine cavity.

Compared with traditional two-dimensional ultrasound, the three-dimensional transvaginal ultrasound under deep learning had obvious advantages in the diagnosis of intrauterine adhesions. However, there were also some shortcomings, such as the limitations when targeting at peripheral intrauterine adhesions and mild adhesions [20, 21]. There appear missed diagnoses in the diagnosis of mild adhesions. Therefore, in the diagnosis of clinically suspected intrauterine adhesion, a more comprehensive observation method is required. In the study, it was found that the intrauterine adhesions and their degrees could be diagnosed more effectively and accurately, as three-dimensional transvaginal ultrasound under deep learning was combined with data analysis of endometrial volume $(\mathrm{V})$ and endometrial blood flow parameters (VI, FI, VFI). There was a positive and effective significance for the development of grading standards for intrauterine adhesions, and a practical theoretical basis for the subsequent diagnosis and treatment of intrauterine adhesions was provided.

\section{Conclusion}

The objective of this study was to use deep learning technology to optimize the three-dimensional transvaginal ultrasound image to diagnose intrauterine adhesion. The results showed that the transvaginal three-dimensional examination image processed by the partial differential algorithm had less noise and clearer structure. However, some limitations in the study should be noted. The sample size is small, which will reduce the power of the study. In the follow-up, expanded sample size is necessary to strengthen the findings of the study. In conclusion, the three-dimensional transvaginal examination can effectively improve the diagnostic efficiency of intrauterine adhesion, providing theoretical support for the subsequent diagnosis and grading of intrauterine adhesion.

\section{Data Availability}

The data used to support the findings of this study are available from the corresponding author upon request. 


\section{Conflicts of Interest}

The authors declare no conflicts of interest.

\section{References}

[1] E. Dreisler and J. J. Kjer, “Asherman's syndrome: current perspectives on diagnosis and management," International Journal of Women's Health, vol. 11, pp. 191-198, 2019.

[2] Z. Khan and J. M. Goldberg, "Hysteroscopic management of asherman's syndrome," Journal of Minimally Invasive Gynecology, vol. 25, no. 2, pp. 218-228, 2018.

[3] X. Santamaria, K. Isaacson, and C. Simón, “Asherman's Syndrome: it may not be all our fault," Human Reproduction, vol. 33, no. 8, pp. 1374-1380, 2018.

[4] C. A. Salazar, K. Isaacson, and S. Morris, "A comprehensive review of Asherman's syndrome: causes, symptoms and treatment options," Current Opinion in Obstetrics and Gynecology, vol. 29, no. 4, pp. 249-256, 2017.

[5] J. Bosteels, S. van Wessel, S. Weyers et al., "Hysteroscopy for treating subfertility associated with suspected major uterine cavity abnormalities," Cochrane Database of Systematic Reviews, vol. 12, no. 12, Article ID CD009461, 2018.

[6] X. Mo, G. Qin, Z. Zhou, and X. Jiang, "Assessment of risk factors of intrauterine adhesions in patients with induced abortion and the curative effect of hysteroscopic surgery," Journal of Investigative Surgery, vol. 32, no. 1, pp. 85-89, 2019.

[7] A. R. Gilman, K. M. Dewar, S. A. Rhone, and M. R. Fluker, "Intrauterine adhesions following miscarriage: look and learn," Journal of Obstetrics and Gynaecology Canada, vol. 38, no. 5, pp. 453-457, 2016.

[8] T. Wang, Y. Zhou, J. Fu, M. Chen, and Y. Luo, "Level of depression, anxiety and stress in patients with intrauterine adhesions in Hunan Province, China: a cross-sectional study," PLoS One, vol. 15, no. 3, Article ID e0229832, 2020.

[9] P. Capmas, A. Mihalache, L. Duminil, L. S. Hor, A.-G. Pourcelot, and H. Fernandez, "Intrauterine adhesions: what is the pregnancy rate after hysteroscopic management?" Journal of Gynecology Obstetrics and Human Reproduction, vol. 49, no. 7, Article ID 101797, 2020.

[10] J. M. Turocy and B. W. Rackow, "Uterine factor in recurrent pregnancy loss," Seminars in Perinatology, vol. 43, no. 2, pp. 74-79, 2019.

[11] G. Centini, L. Troia, L. Lazzeri, F Petraglia, and S Luisi, "Modern operative hysteroscopy," Minerva Ginecologica, vol. 68, no. 2, pp. 126-132, 2016.

[12] M. J. Kim, Y. Lee, C. Lee et al., "Accuracy of three dimensional ultrasound and treatment outcomes of intrauterine adhesion in infertile women," Taiwanese Journal of Obstetrics \& Gynecology, vol. 54, no. 6, pp. 737-741, 2015.

[13] F. Ahmadi and M. Javam, "Role of 3D sonohysterography in the investigation ofuterine synechiae/asherman's syndrome: p," Journal of Medical Imaging and Radiation Oncology, vol. 58, no. 2, pp. 199-202, 2014.

[14] S. Guo, R. Chen, H. Li, and T. Zhang, "Identify severity bug report with distribution imbalance by CR-SMOTE and ELM," International Journal of Software Engineering and Knowledge Engineering, vol. 29, no. 2, pp. 139-175, 2019.

[15] M. Prior, A. Richardson, S. Asif et al., "Outcome of assisted reproduction in women with congenital uterine anomalies: a prospective observational study," Ultrasound in Obstetrics and Gynecology, vol. 51, no. 1, pp. 110-117, 2018.

[16] S. Baradwan, A. Baradwan, and D. Al-Jaroudi, "The association between menstrual cycle pattern and hysteroscopic march classification with endometrial thickness among infertile women with Asherman syndrome," Medicine, vol. 97, no. 27, Article ID e11314, 2018.

[17] A. Ludwin, I. Ludwin, M. Kudla et al., "Diagnostic accuracy of three-dimensional sonohysterography compared with office hysteroscopy and its interrater/intrarater agreement in uterine cavity assessment after hysteroscopic metroplasty," Fertility and Sterility, vol. 101, no. 5, pp. 1392-1399, 2014.

[18] J. N. Karim, N. W. Roberts, L. J. Salomon, and A. T. Papageorghiou, "Systematic review of first-trimester ultrasound screening for detection of fetal structural anomalies and factors that affect screening performance," Ultrasound in Obstetrics and Gynecology, vol. 50, no. 4, pp. 429-441, 2017.

[19] G. Pados, A. Daniilidis, J. Keckstein, P Papandreou, and S Gordts, "A European survey on the conservative surgical management of endometriotic cysts on behalf of the European Society for Gynaecological Endoscopy (ESGE) Special Interest Group (SIG) on Endometriosis," Facts, views \& vision in ObGyn, vol. 12, no. 2, pp. 105-108, 2020.

[20] P. Szkodziak, S. Woźniak, P. Czuczwar et al., "Usefulness of three dimensional transvaginal ultrasonography and hysterosalpingography in diagnosing uterine anomalies," Ginekologia Polska, vol. 85, no. 5, pp. 354-359, 2014.

[21] L. Pleş, C. Alexandrescu, C. A. Ionescu, C. A Arvătescu, S Vladareanu, and M. A Moga, "Three-dimensional scan of the uterine cavity of infertile women before assisted reproductive technology use," Medicine, vol. 97, no. 41, Article ID e12764, 2018. 